\title{
Mechanical resonance of the austenite/martensite interface and the pinning of the martensitic microstructures by dislocations in $\mathrm{Cu}_{74.08} \mathrm{Al}_{23.13} \mathrm{Be}_{2.79}$
}

\author{
E. K. H. Salje, ${ }^{1}$ H. Zhang, ${ }^{1}$ H. Idrissi, ${ }^{2}$ D. Schryvers, ${ }^{2}$ M. A. Carpenter, ${ }^{1}$ X. Moya, ${ }^{3}$ and A. Planes ${ }^{4}$ \\ ${ }^{1}$ Department of Earth Sciences, University of Cambridge, Downing Street, Cambridge CB2 3EQ, United Kingdom \\ ${ }^{2}$ EMAT, University of Antwerp, Groenenborgerlaan 171, B-2020 Antwerp, Belgium \\ ${ }^{3}$ Department of Material Sciences, University of Cambridge, Pembroke Street, Cambridge CB2 3QZ, United Kingdom \\ ${ }^{4}$ Departament d'Estructura i Constituents de la Matèria, Facultat de Física, Universitat de Barcelona, \\ Diagonal 647, 08028 Barcelona, Spain
}

(Received 12 March 2009; revised manuscript received 10 June 2009; published 16 October 2009)

\begin{abstract}
A single crystal of $\mathrm{Cu}_{74.08} \mathrm{Al}_{23.13} \mathrm{Be}_{2.79}$ undergoes a martensitic phase transition at 246 and $232 \mathrm{~K}$ under heating and cooling, respectively. The phase fronts between the austenite and martensite regions of the sample are weakly mobile with a power-law resonance under external stress fields. Surprisingly, the martensite phase is elastically much harder than the austenite phase showing that interfaces between various crystallographic variants are strongly pinned and cannot be moved by external stress while the phase boundary between the austenite and martensite regions in the sample remains mobile. This unusual behavior was studied by dynamical mechanical analysis (DMA) and resonant ultrasound spectroscopy. The remnant strain, storage modulus, and internal friction were recorded simultaneously for different applied forces in DMA. With increasing forces, the remnant strain increases monotonously while the internal friction peak height shows a minimum at 300 $\mathrm{mN}$. Transmission electron microscopy shows that the pinning is generated by dislocations which are inherited from the austenite phase.
\end{abstract}

DOI: 10.1103/PhysRevB.80.134114

PACS number(s): 62.20.fg, 62.40.+i, 64.70.kd

\section{INTRODUCTION}

Martensitic phase transformations lead to shape memory effects, superelasticity, and, sometimes, high mechanical damping in alloys (e.g., Refs. 1 and 2). Two types of stressinduced interfacial properties commonly dominate the behavior of martensites. ${ }^{3-13}$ First, the phase front between coexisting austenite and martensite regions in a sample are generally mobile. The location of the phase front depends sensitively on thermal gradients, boundary conditions, and defect configurations. Song et al. ${ }^{9,14}$ have carefully examined the friction properties of the phase front between the austenite and martensite regions of the sample with similar composition. We will argue that their results are fully confirmed by our experiments using a different experimental technique; their analysis of the aging properties is also in agreement with our observations. We find, indeed, that phase fronts are mobile but strongly dissipative. The dissipation changes with the decoration of the interfaces with defects. However, our sample shows a very different behavior for the second type of stress-related movement shifts of interfaces, namely, those between different crystallographic variants of the martensite phase. These interfaces are often twin related ${ }^{15}$ and have been studied extensively in ferroelastic samples. ${ }^{16-23}$ Not all interfaces between the martensite variants are experimentally mobile but, usually, some of them are. A typical example is $\mathrm{Cu}_{69.1} \mathrm{Zn}_{18.5} \mathrm{Al}_{12.3}$ where highly mobile interfaces exist together with immobile interfaces (Ref. 15, composition after conversion into atomic \%). The overall observation is that the martensitic phase is elastically much softer than the austenitic phase because some or all interfaces move under external stress. Even more so, such effects are observed in ferroelastic oxides, perovskites, fluorites, and sulfates. ${ }^{17-23}$ In these materials pinning is very weak in ferroelastic fluorites ${ }^{17}$ while damping does not exist in virtually defect-free materials such as $\mathrm{SrTiO}_{3}{ }^{18}$ Resonance damping was found in materials with oxygen defects such as $\mathrm{LaAlO}_{3} .{ }^{16}$

In this paper we will argue that $\mathrm{Cu}_{74.08} \mathrm{Al}_{23.13} \mathrm{Be}_{2.79}$ displays a very different behavior. Instead of softening in the martensite phase the material becomes effectively harder. This could either mean that martensite-martensite interfaces do not exist or that such interfaces are heavily pinned. Some interfaces were observed in our transmission electron microscopy (TEM) experiments and we will argue that pinning of these interfaces is the most likely mechanism for the observed mechanical hardening of the martensitic phase.

A similar effect was previously observed in $\mathrm{Cu}-\mathrm{Al}-\mathrm{Ni}^{24}{ }^{24} \mathrm{It}$ was reported there that the hardening was the same for samples which were aged to different degrees. In $\mathrm{Cu}_{74.08} \mathrm{Al}_{23.13} \mathrm{Be}_{2.79}$ one could argue that the required strong pinning could be provided by decomposition of the sample ${ }^{25}$ while TEM observations indicate that pinning happens via dislocations which nucleate during the martensitic phase transformation on dislocations inherited from the austenite. Planes et al. ${ }^{26}$ have argued that in $\mathrm{Cu}_{74.08} \mathrm{Al}_{23.13} \mathrm{Be}_{2.79}$ the elastic strain and interfacial energies are relevant and may contribute to the latent heat of the transformation. Our observations confirm this idea but in a rather unexpected way: the pinning of interfaces relate to jamming by dislocations which nucleate at the transition point. The role of mobile impurities for the pinning and unpinning of dislocations was previously invoked by Gonzalez et al. ${ }^{27}$ for the aging behavior of a similar sample.

In order to explore the resonance features of the mechanical behavior in $\mathrm{Cu}_{74.08} \mathrm{Al}_{23.13} \mathrm{Be}_{2.79}$ we employ two different techniques. The low-frequency part of the spectra $(0.25-10$ $\mathrm{Hz})$ relate to movements of interfaces or bending of large parts of them. The appropriate instrument is the DMA 
(dynamical mechanical analyzer) which also allows to test the rupture stress of interfaces using static bias stress superimposed with the dynamical part of the mechanical excitation. ${ }^{20,28,29}$ High frequencies and low amplitudes yield information about the local mobility of interfaces such as local kinks and short-wavelength ripples. The methodology here is the RUS (resonant ultrasonic spectroscopy) at frequencies in the $\mathrm{MHz}$ region. ${ }^{30}$

\section{EXPERIMENTAL DETAILS}

The atomic composition of the sample is $\mathrm{Cu}_{74.08} \mathrm{Al}_{23.13} \mathrm{Be}_{2.79}$. as determined by microprobe analysis of $\mathrm{Cu}$ and $\mathrm{Al}$ while the Be content was inferred from the composition of the melt. From the original rod, a sample with length $7.2 \mathrm{~mm}$, width $3.90 \mathrm{~mm}$, and thickness $0.15 \mathrm{~mm}$ for dynamical mechanical analysis measurement was cut using a low-speed diamond saw. The surface of the sample was parallel to (100) and its length parallel to [110]. A small additional piece was cut for differential scanning calorimetric (DSC) measurement.

The DSC experiments were performed using a highsensitivity calorimeter at a heating/cooling rate of approximately $0.5 \mathrm{~K} / \mathrm{min}$. Mechanical properties were measured using a Perkin-Elmer dynamical mechanical analyzer (DMA$7 \mathrm{e})$ operating in three-point bend geometry. The sample was suspended on two knife edges, $5 \mathrm{~mm}$ apart. A force is applied from above via a third knife edge located halfway between the supporting knives. The total force is the sum of a static component $F_{S}$ and a dynamic component with amplitude $F_{D}$ and frequency $f$. The amplitude of deflection $\left(u_{D}\right)$ and phase lag $(\delta)$ are measured with resolutions of $\Delta u_{D}$ around $10 \mathrm{~nm}$ and $\Delta \delta=0.1^{\circ}$, respectively. The dynamic young's modulus is then

$$
E=\frac{l^{3}}{4 t^{3} w} \frac{F_{D}}{u_{D}} \exp (i \delta),
$$

where $l, w$, and $t$ are the distance between knife edges, the width, and the thickness of the sample, respectively. The real $\left(E^{\prime}=|E| \cos (\delta)\right)$ and imaginary $\left(E^{\prime \prime}=|E| \sin (\delta)\right)$ components of the dynamic modulus are referred to as the storage and loss modulus, respectively. The ratio $E^{\prime \prime} / E^{\prime}=\tan (\delta)$ is a measure for the internal friction. The probe position is evaluated at the mid point of the sample's top surface.

Two different types of experiments have been performed: temperature scans and frequency scans. The temperature scans were performed with various dynamic forces at a frequency of $f=4 \mathrm{~Hz}$ while the temperature was swept between room temperature and $120 \mathrm{~K}$ with a rate of $\dot{T}=5 \mathrm{~K} / \mathrm{min}$. The dynamical force was programmed to maintain $F_{S}=1.1 F_{D}$ at all times.

The RUS experiments were performed to check whether the pinning effects are strongly frequency dependent. The same sample as in the DMA experiments was used. Previous RUS work used a polycrystalline sample with different composition and found softening of the elastic moduli in the martensite phase. ${ }^{30}$ We will show that no significant frequency dispersion exists in the range between $2 \mathrm{~Hz}$ and 1 $\mathrm{MHz}$.

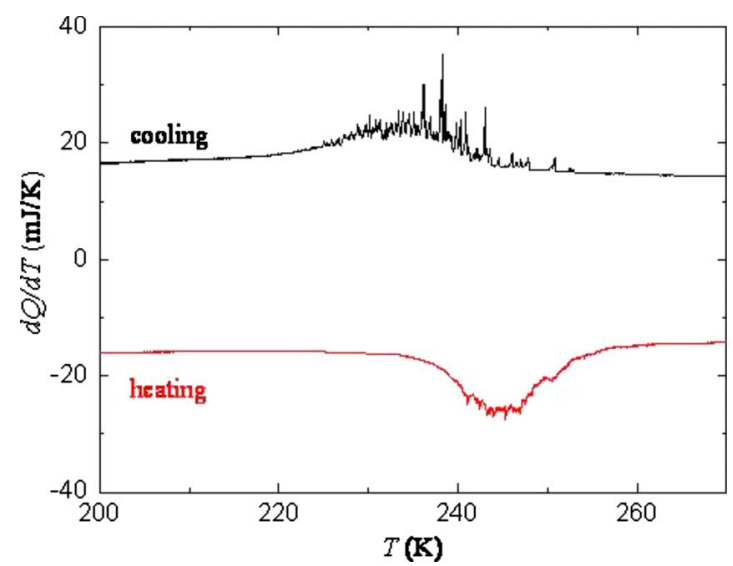

FIG. 1. (Color online) DSC curves under cooling and heating.

For the TEM study, a sample with a slightly different composition was used in order to allow for the martensitic transformation to occur around room temperature and to observe austenite and martensite regions at room temperature in a single sample. A Philips $\mathrm{LaB}_{6} \mathrm{CM} 20$ TEM operated at $200 \mathrm{kV}$ was used. The microstructure of the martensite phase in the as-received material was analyzed using a classical double tilt holder at room temperature while a double-tilt liquid nitrogen cooling holder was used to investigate the evolution of the microstructure during cooling.

Crystallographic defects were studied using the "twobeam" bright field (BF) and the dark field (DF) techniques, based on the diffraction contrast imaging theory. In addition, the weak-beam dark field (WBDF) technique was used to elucidate dislocation near-core properties. TEM samples were prepared by a routine procedure of mechanical grinding with subsequent double-jet electropolishing at $278 \mathrm{~K}$ using a voltage of $12 \mathrm{~V}$. A $500 \mathrm{ml}$ distilled water, $250 \mathrm{ml}$ ethyl alcohol, $250 \mathrm{ml}$ orthophosphoric acid, $50 \mathrm{ml}$ propyl alcohol, and $5 \mathrm{~g}$ urea solution were used as electrolyte.

\section{RESULTS AND DISCUSSION}

\section{Thermal and mechanical properties}

The transformation between austenite and martensite is clearly visible in the results of calorimetric measurements in Fig. 1. The austenite phase has the cubic $\beta$ structure while the martensitic phase has a monoclinic long-period structure which is usually indicated as $18 \mathrm{R}$. When cooled from high temperatures the sample first undergoes an order/disorder transition above $800 \mathrm{~K}$ to a DO3 structure and transforms martensitically at low temperatures. The complex transition mechanism relates to these two phases. Song et al. ${ }^{14}$ have argued that even under rapid quench the sample transforms via an intermediate phase $\beta-\mathrm{DO}_{3}-18 \mathrm{R}$ while the equilibrium phases are fcc (a) and $\gamma_{2}$. In our experiments we see only one transition step within a broad temperature range (up to $800 \mathrm{~K}$ ). This step is somewhat broadened and occurs at $232 \mathrm{~K}$ under cooling and at $246 \mathrm{~K}$ under heating, respectively (Fig. 1). Note the jerky character of the transformation which is most likely influenced by avalanches. 

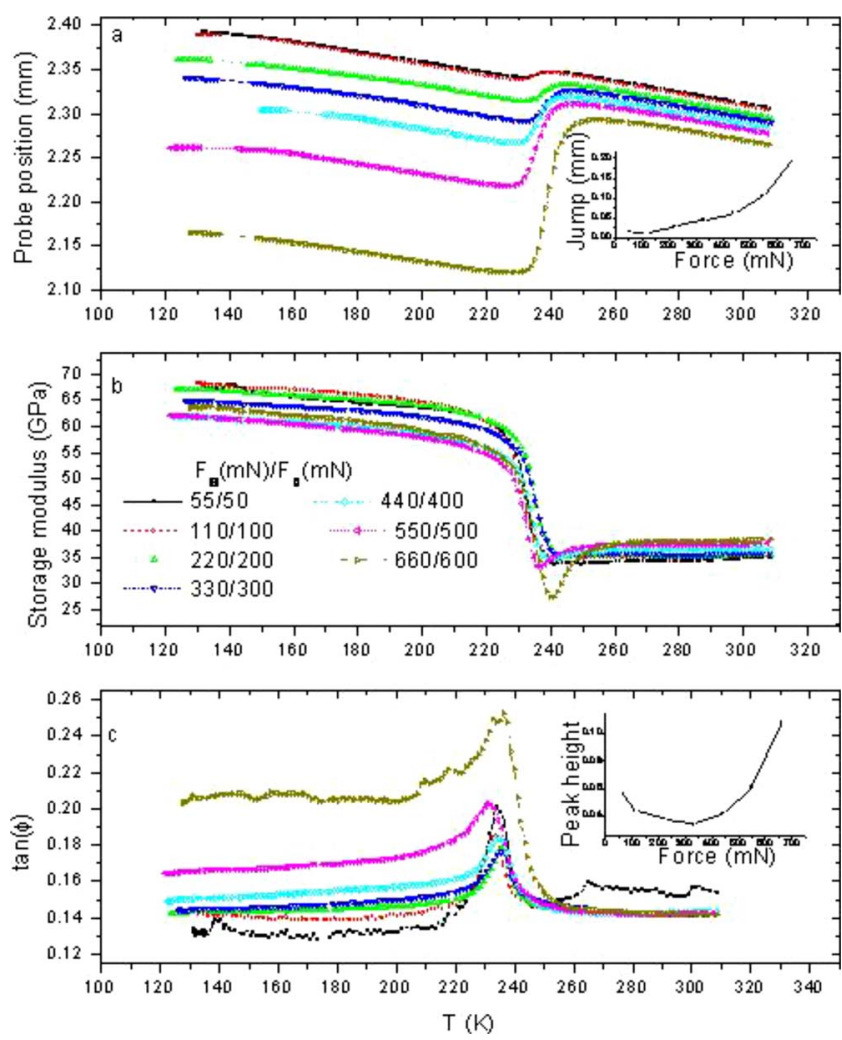

FIG. 2. (Color online) Temperature dependence of the probe position, storage modulus, and internal friction in the heating process with ramp rate of $\dot{T}=5 \mathrm{~K} / \mathrm{min}$, frequency $f=4 \mathrm{~Hz}$ for various forces. The force dependence of the jump was inserted in (a); the force dependence of the peak height of the internal friction is inserted in (c), all forces are measured in $\mathrm{mN}$.

Figure 2 shows the temperature dependence of the probe position, storage modulus, and internal friction $\tan (\delta)$ at heating rate of $\dot{T}=5 \mathrm{~K} / \mathrm{min}$ and a frequency of $f=4 \mathrm{~Hz}$ with various dynamic forces. The probe position shows a jump at the transformation region while outside this region it changes linearly as a function of temperature. The jump reflects the shape change in the sample during the martensitic transformation, equivalent to the spontaneous strain in ferroelastic materials.22 The force dependence stems from the increasing number of martensitic variants which nucleate in the optimal direction when the transformation happens under increasing bias field. The smaller change in the probe position in the austenite phase is simply related to the bending of the sample but not to any structural features.

The storage modulus exhibits a minimum near the phasetransition region for all bias forces. For the applied force larger than $300 \mathrm{mN}$, this minimum is clearly seen as a dip of the modulus just before the hardening occurs in the martensite phase. This dip increases with increasing bias force.

The friction parameter $\tan (\delta)$ shows a peak where the dip in the storage modulus appears. This peak becomes larger and more asymmetric for larger bias forces. The movement of the interphase boundaries is hence enhanced by the bias force via the unpinning of previously static boundaries. The higher number of mobile interphase boundaries leads then to a higher friction parameter. This scenario is typical for the depinning of interfaces (rupture) by external forces ${ }^{20}$ and shows that many but not all interphase boundaries are already pinned during the phase transformation. The inset of Fig. 2 (bottom) shows that the rupture bias force is approximately $300 \mathrm{mN}$. Our results agree well with and expand those of Song et al..$^{14}$

To separate the various pinning mechanisms for the interfaces between austenite and martensite we performed experiments where smaller (larger) force was applied to the sample under cooling to get a smaller (larger) remnant strain while in the heating process larger (smaller) force was applied to enhance (reduce) the stress-dependent effect. Figure 3(a) shows the results with an applied force of $200 \mathrm{mN}$ under cooling and a subsequent heating process with $500 \mathrm{mN}$. In Fig. 3(b) the results with an applied force of $500 \mathrm{mN}$ under cooling and a consequent heating process with $200 \mathrm{mN}$ are shown. In both cases the remnant strain is determined by the force applied in the initial cooling process, as the jump at the transition point is the same in the cooling and heating process even though the bias forces are different. The deformation of the probe is, thus, determined by the orientation of the martensitic variants under external field at the transition temperature when the sample is cooled into the martensite state. No further changes occur when the bias field is subsequently changed at low temperatures.

The bias effect is small for the storage modulus with the expected increase in the dip when the temperature is lowered under a stronger bias (i.e., the interface is mobilized under the bias field). This is also seen in the loss angle while a small bias leads to small losses at the transformation point we find stronger losses once the local interfaces are depinned by the bias. Losses are always higher in the martensite phase when the sample is cooled under high mechanical bias then heated under a smaller bias (Fig. 3).

The spectra discussed show very little frequency dispersion. The only exception is the frequency dependence of the internal friction peak in Fig. 4. In order to analyze the friction in more detail experiments were performed at frequencies between 0.25 and $10 \mathrm{~Hz}$. The upper part of the graph in Fig. 4 shows clearly that the storage modulus is frequency independent while the loss angle in the lower part shows an increase with decreasing frequency. This behavior is also observed for the integrated peak area (i.e., the total loss over the entire transformation regime): a strong increase occurs below approximately $2 \mathrm{~Hz}$. In Fig. 5 the experimental data are compared with a power-law decay of $f^{-0.2}$.

The high-frequency measurements using RUS essentially confirm the DMA data. The RUS experiments allow the separation of the two major contributions of the elastic moduli $C_{44}$ and $C_{11}-C_{12}{ }^{15}$ Figure 6 shows an illustrative section of RUS spectra collected during heating. Individual resonance peaks can be followed through the phase transition and all show the same qualitative trend of increasing frequency with falling temperature through the martensitic transition, confirming the trend of elastic stiffening observed at low frequencies by DMA. Some peaks show again a distinct softening above of the transition with a large stiffening below it while others show slight stiffening in advance of the transition and a smaller frequency shift at lower temperatures. These must correspond to the limiting cases of distor- 

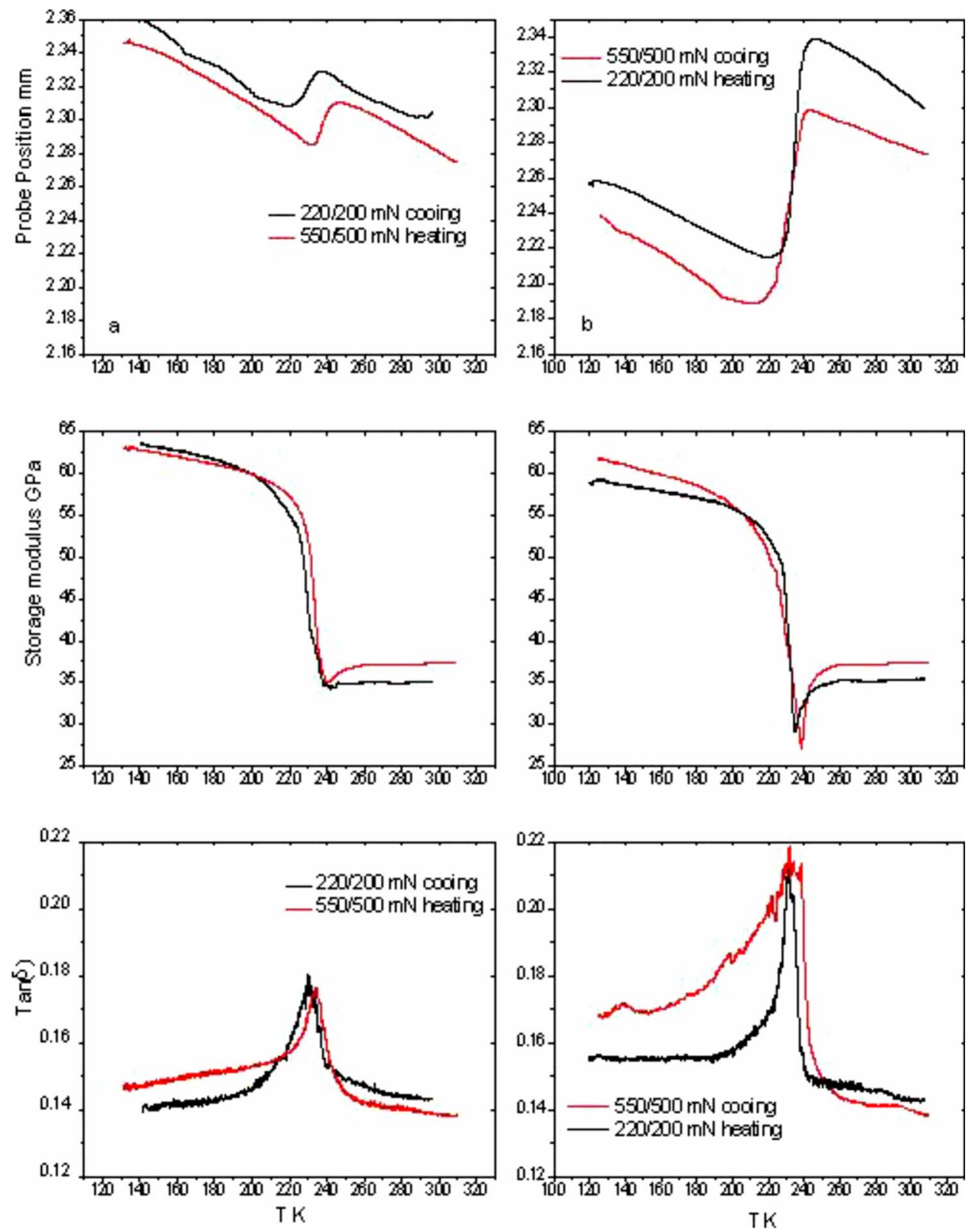

FIG. 3. (Color online) Temperature dependence of the probe position, storage modulus, and internal friction at ramp rate of $5 \mathrm{~K} / \mathrm{min}$ (a) $200 \mathrm{mN}$ under cooling and $500 \mathrm{mN}$ under heating and (b) $500 \mathrm{mN}$ under cooling and $200 \mathrm{mN}$ under heating.

tion modes determined predominantly by $C_{44}$ or $\frac{1}{2}\left(C_{11}-C_{12}\right)$, as the variations in frequency of all the other peaks could be made up of a combination of the two in different proportions. With falling temperature ahead of the phase transition $C_{44}$ increases slightly while $\frac{1}{2}\left(C_{11}-C_{12}\right)$ decreases ${ }^{26}$ thus allowing a tentative assignment of the RUS peaks. Two peaks indicated in Fig. 6, representing the limiting cases described above, were selected for detailed analysis. At $287 \mathrm{~K}$ these have resonance frequencies of 64.78 and $283.81 \mathrm{kHz}$, and were scaled to $C_{44}=94.9 \mathrm{GPa}$ and $\frac{1}{2}\left(C_{11}-C_{12}\right)=7.15 \mathrm{GPa},{ }^{26}$ respectively. The same scaling was then applied to measured frequencies through the entire cooling and heating cycle. The results are shown in Fig. 7.

\section{MICROSTRUCTURAL PROPERTIES}

\section{A. Dislocations inherited from austenite}

Figure 8 shows a martensite plate inside an austenite matrix imaged under BF two-beam conditions using the martensite $1 \overline{2} \overline{8}$ reflection. The $18 \mathrm{R}$ structure of the martensite is clear from the row of superlattice reflections in the $[\overline{2} \overline{1} 0]$ selected area diffraction pattern (SADP) shown in the inset and originating from the encircled region in the martensite plate. The $c$ direction of the martensite shows a small deviation from the $[-110]$ direction of the austenite while the [100] and [001] axes of the martensite correspond with the [110] and [001] directions in the austenite, respectively. In this image, a high density of dislocations in the austenite 


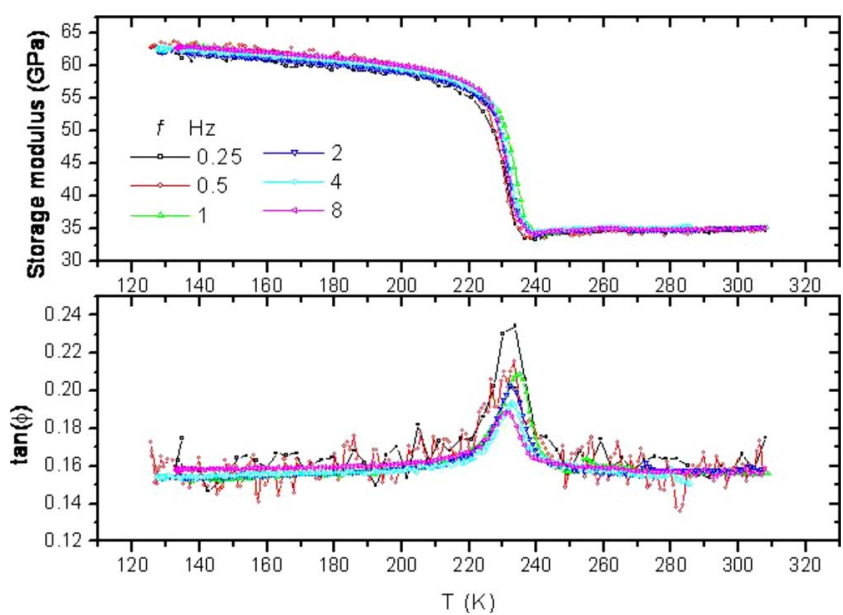

FIG. 4. (Color online) Temperature dependence of storage modulus and internal friction with various frequencies in the heating process with $F_{D} / F_{S}=200 / 220 \mathrm{mN}$ and $\dot{T}=5 \mathrm{~K} / \mathrm{min}$.

matrix regions, both left and right from the martensite plate, can be observed. ${ }^{31}$ Some of these dislocations, in particular, those with dislocation lines close to perpendicular to the trace of the austenite-martensite interface, are seen to propagate from the austenite into the martensite plate through the austenite-martensite interface. These dislocations will consequently affect the plasticity of the martensite. A second family reveals dislocation lines close to parallel to the trace of the austenite-martensite interface.

Figure 9(a) is a WBDF image obtained using the 0018 reflection corresponding with the basal or close-packed planes of the martensite. This image reveals contrast of two populations of dislocations, types $\mathrm{A}$ and $\mathrm{B}$, inside the martensite plate. It can be expected that the glide planes for populations A and B correspond to (128) and (0018) planes of the martensite structure, respectively, since the dislocation lines are parallel to these planes. From Fig. 8 a homogeneous distribution of type A dislocations along the martensite plate

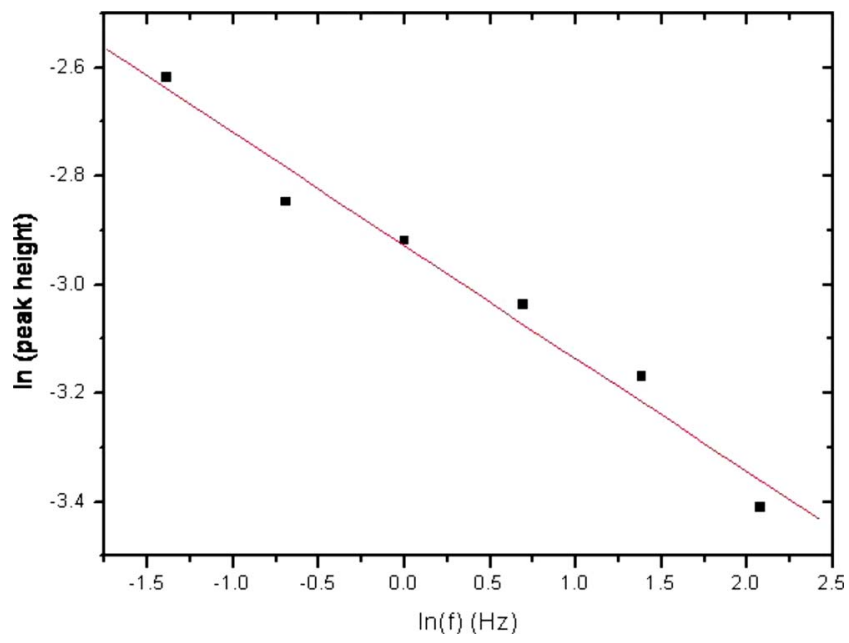

FIG. 5. (Color online) Double-logarithmic plot of the frequency dependence of the height of the loss peak in Fig. 4. The decay is consistent with $\tan (\delta)=A f^{-0.2}$.

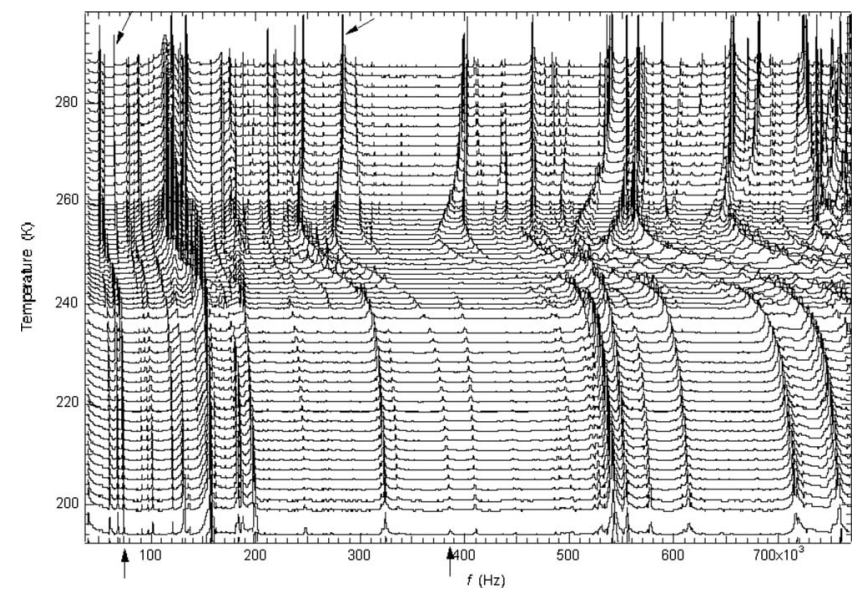

FIG. 6. A section of RUS spectra collected during heating. The left-hand axis is really amplitude but the spectra have been shifted in proportion to the temperature at which they were collected and the temperature scale is shown instead. The two pairs of arrows (two low and two high-temperature peaks) indicate the two resonances which were analyzed in detail.

compared to type B dislocations can be noted. In addition, in Fig. 9, an intersection between an A and a B dislocation is indicated by a black arrow. At the intersection point, the deviation of the line orientation of the $\mathrm{B}$ type dislocation is seen. The origin of these dislocations can be deduced by carefully analyzing the image of Fig. 9(a). Dislocations of type $\mathrm{A}$ in the martensite are seen to originate from the austenite at spots marked by white arrows. The small orientation deviation exhibited by these dislocations when crossing the interface is probably due to the orientation difference between the glide planes in both phases. Also, although no type $\mathrm{B}$ dislocations are seen to cross the austenite-martensite interface, Fig. 8 clearly shows a family of dislocations in the austenite again parallel with the martensite type B ones. In

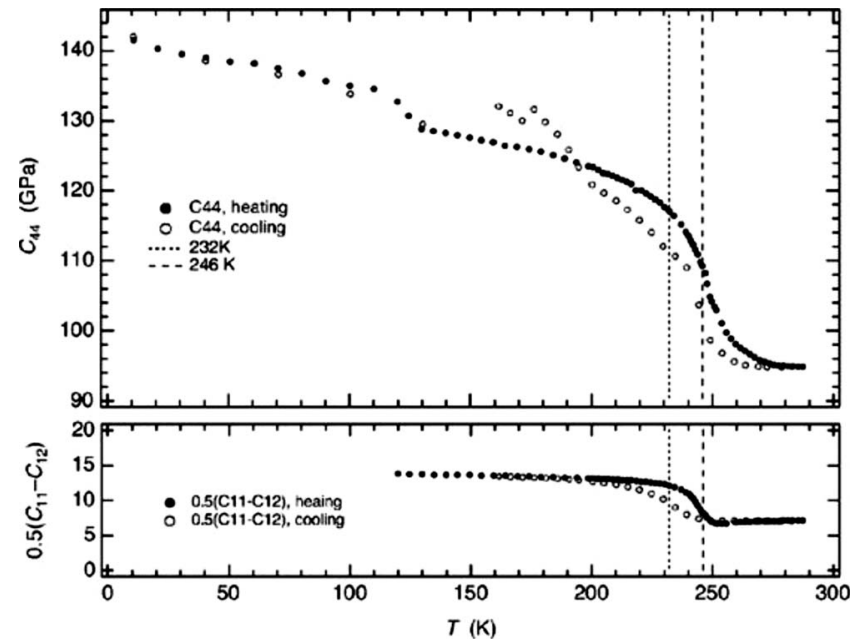

FIG. 7. Variations in elastic constants obtained from fitting to resonance peaks with frequencies of (a) $\sim 284$ and (b) $\sim 65 \mathrm{kHz}$ at room temperature. Broken lines represent transition temperatures taken from the DSC analysis. The hardening in the martensitic phase is clearly visible. 


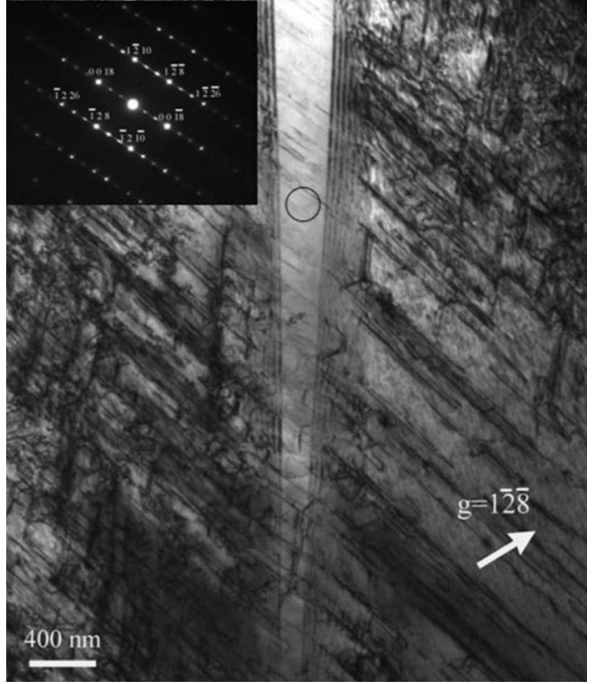

FIG. 8. Two-beam BF micrograph using $g=1 \overline{2} \overline{8}$ showing a martensite plate inside the austenite matrix revealing an abundance of dislocations. The latter can roughly be divided into two groups, those with dislocation lines close to perpendicular to and those with dislocation lines close to parallel with the trace of the austenitemartensite interface. The inset shows a SADP of the $18 \mathrm{R}$ martensite along $[\overline{2} \overline{1} 0]$ originating from the encircled region in the martensitic plate.

other words, it is assumed that also the observed type B dislocations in the martensite originate from the latter family of dislocations in the austenite. In Fig. 9(b), the close-packed stacking of the $18 \mathrm{R}$ structure becomes visible as a result of the imaging contrast of many parallel stacking defects. Halfway the plate, and indicated by a white arrow, some of these stacking defects are seen to stop at a dislocation running

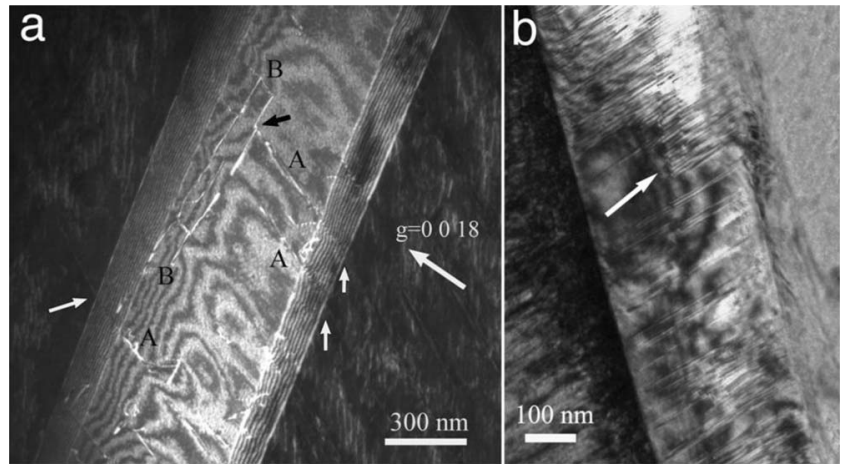

FIG. 9. (a) WBDF micrograph obtained with $g=0018$ showing both types of martensite dislocations inherited from the austenite. Type A dislocations are seen as sharp white lines close to perpendicular to the trace of the austenite-martensite interface while type $\mathrm{B}$ dislocations are sharp white lines close to parallel with that interface trace (not to be mistaken with the fringes on the edge of the plate and due to the overlapping of the matrix and the plate). The black arrow points at an intersection of types A and B dislocation while the white arrows indicate intersection points of the type A dislocations with the austenite-martensite interface. (b) Stacking defects of the $18 \mathrm{R}$ stacking are seen to stop at a dislocation inside a martensite plate (white arrow).

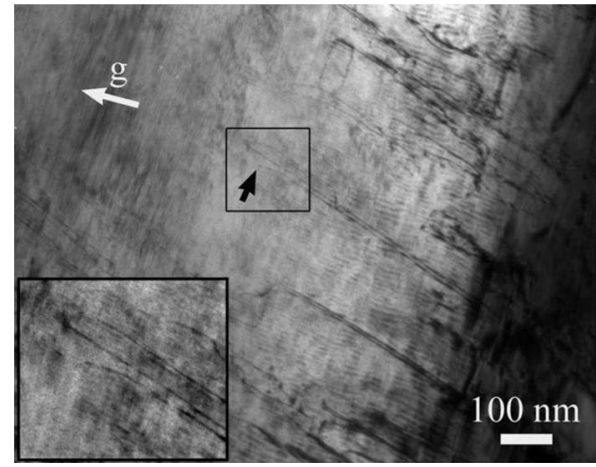

FIG. 10. Two-beam BF micrograph obtained with $g=0018$ revealing the dissociation of the type A dislocations as well as an increase in dissociation distance at the end of the dislocation line.

close to parallel with the long axis of the martensite plate, although the present imaging conditions are not ideal for visualizing the dislocation line.

Figure 10 shows a two-beam BF micrograph using the same 0018 reflection (i.e., similar to the image of the Fig. 9) but now the sample was tilted away from the $[\overline{2} \overline{1} 0]$ zone axis in order to verify if the type A dislocations correspond to undissociated perfect dislocations or to partial dislocations (PDs) bounding stacking faults (SFs). From this image each type A dislocation reveals a clear dissociation into two partial dislocations. The average dissociation distance was found to be around $15 \mathrm{~nm}$. In Fig. 10, SF fringes between the leading and the trailing partial dislocations are not visible but in the region marked by the black arrow an increase in the dissociation distance at the end of the dislocation line can be observed, which is characteristic of a typical surface effect for dissociated dislocations. It should further be noted that the nanoscale size of the studied martensite plate makes the analysis of the dislocation morphology and the identification of the Burgers vector of the dislocations inside the martensite impossible. In the literature, stacking faults on $\{12 \overline{8}\}$ type planes were observed in $18 \mathrm{R}$ martensite of $\mathrm{Cu}-\mathrm{Al}-\mathrm{Ni}$ alloys. ${ }^{32,33}$ Recently, screw dislocations on the (128) plane and which do not extend into partials were reported by Zhang et al. ${ }^{34}$ in a deformed $18 \mathrm{R}$ martensite $\mathrm{Cu}-\mathrm{Zn}-\mathrm{Al}$ and $\mathrm{Cu}-\mathrm{A} 1$ shape memory alloy.

For the dislocations of type B, which are parallel to the (0018) glide plane, it can be expected that they correspond to PD bounding a SF since the contrast of these dislocations in Fig. 9 show a discontinuous contrast (also observed for type A dislocations). This phenomenon is due to the superposition of the contrast of the partial dislocations and the SF fringes when the SF plane is parallel to the electron beam. In the literature, such dislocations gliding in the (001) ${ }_{18 \mathrm{R}}$ plane were reported by Rodriguez et al. ${ }^{35,36}$ in plastically deformed $18 \mathrm{R}$ martensite of $\mathrm{Cu}-\mathrm{Zn}-\mathrm{Al}$ alloys. The total Burgers vector of the dislocations is $b=[010]_{18 \mathrm{R}}$. The authors observed that the Burgers vector first dissociates into two partials with $b_{0}$ $=\frac{1}{2}[010]$. Each one of these partials will further dissociate into two Shockley partials with $b_{1}=\frac{1}{12}[230]$ and $b_{2}$ $=\frac{1}{12}[\overline{2} 30]$.

In very few cases the martensite plates revealed the expected twinning as seen in Fig. 11(a), where the twin char- 


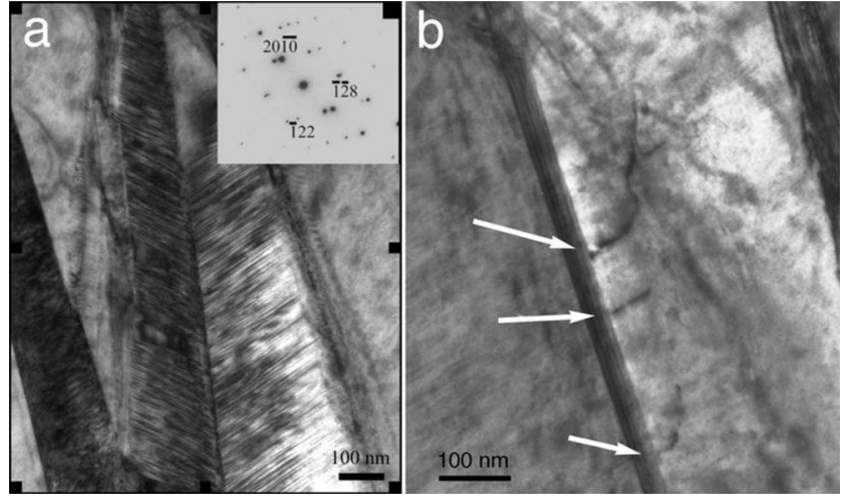

FIG. 11. (a) Martensite plate showing a twin midrib with the corresponding SADP as inset revealing the respective spot splitting. (b) Dislocation lines arriving at the twin midrib of the martensite plate.

acter is clear from the orientation of the stacking on both sides of the midrib as well as from the spot splitting in the SADP inset. The reason for the low fraction of twinning is probably the fact that the martensitic transition has been captured in a very early stage so that the total driving force for introducing twins as stress-relief mechanism is not yet very large. In Fig. 11(b) the same region has been oriented in such a way as to visualize dislocation lines in one of both martensite variants and reaching the midrib or twin interface as indicated by white arrows.

\section{B. Defects introduced by cooling}

A cooling experiment was then carried out in order to study the influence of the dislocations present in the martensite on the microstructure of the plate during the cooling. Figure 12 shows a two-beam DF image obtained in the martensite with a $\overline{1} 210$ reflection after cooling at $-20{ }^{\circ} \mathrm{C}$. In this image, nearly vertical bright lines can be observed which correspond to the population of type A dislocations. In the same image, close to horizontal bright lines parallel to the $c$ plane of the $18 \mathrm{R}$ structure can also be clearly seen. During the in situ cooling experiment, dislocations of type A or B actively crossing the austenite-martensite interface were not

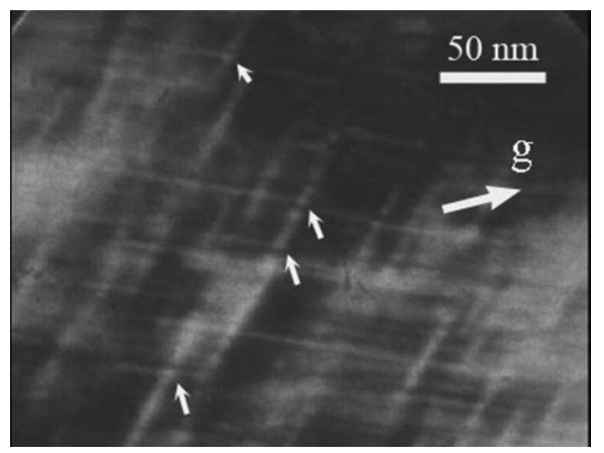

FIG. 12. Two-beam DF micrograph obtained at $-20{ }^{\circ} \mathrm{C}$ with $g=1 \overline{2} 10$ showing an enhanced strain contrast where transformation dislocations tangle with type A dislocations.

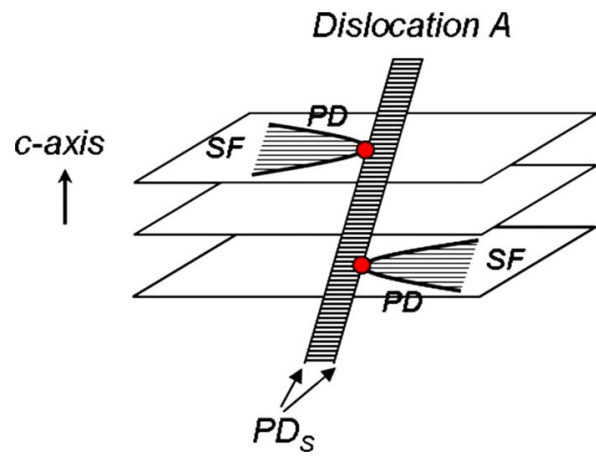

FIG. 13. (Color online) Scheme of the pinning of the PD by a type A dislocation during cooling.

observed. On the other hand, the horizontal bright lines were rapidly crossing the viewing screen inside the martensite plate. It can thus be concluded that these horizontal bright lines in Fig. 12 are intrinsic to the martensitic structure. These new defects probably correspond to partial dislocations originating from the imperfections of the $18 \mathrm{R}$ stacking and are dragging large SF behind them, such as the defects already noticed in Fig. 9(b). In this case, the large SF area is created by the formation and the glide of only the leading PD without the trailing one. The small arrows indicate some intersection points between the expected PD and type A dislocations. At the intersection point the brightness increases due to a local increase in the strain at the intersection point. In some cases, the moving PD is even seen to stop at the type A dislocation, which corresponds to the still image in Fig. 9(b).

Figure 13 shows a scheme which illustrates the interaction between PD in the martensite dragging large SF behind them with the extended dislocations of type A originating from the austenite phase. According to this scheme, during the cooling experiment, PD bounding SF will rapidly pass through the product phase on different close-packed planes. The driving force for the SF expansion is a combination of the local stress in the martensite plate and the phase transformation, which tends to produce a new and energetically more stable structure. The PD at the tip of the SF will be pinned when intersecting the type A dislocations. Although the result of the dislocation interaction depends on the geometry of the interaction and dislocation type, we can expect in the present case that the pinning effect of the extended dislocations of type A on the PD involved in the phase transformation will be more important compared to a perfect dislocation in A due to the elastic strain field of the two dissociated PD and the additional stress at the SF at type A.

Since no moving twin interfaces could be observed, essentially due to experimental limitations such as limited range of tilt, thin-film effect hampering the martensitic transformation and the fact that only very few martensite plates were observed in the first place, an actual live interaction between dislocations and twin interfaces could not be captured. Nevertheless, since the movement of a twin interface occurs in the same way as that of a single SF, it can readily be assumed that the same type of dislocations will strongly interact and possibly pin the twin planes in the same way as they do the $18 \mathrm{R}$ stacking faults. 


\section{SUMMARY}

The elastic response in $\mathrm{Cu}_{74.08} \mathrm{Al}_{23.13} \mathrm{Be}_{2.79}$ is unusual in so far as only the interface between the austenite and the martensite phases are somewhat mobile while the interfaces between the martensite variants are immobile. The pinning of these interfaces relates to a complex interplay between the martensitic phase transformation and the generation of dislocations. The images of the austenite phase show already a multitude of dislocations. This is unusual for untreated shape memory alloys such as in the Ni-Ti or Ni-Ti systems. These dislocations persist in the martensite phase. For some families of dislocations, their orientation is nearly perpendicular to the basal plane of the $18 \mathrm{R}$ structure. Additional basal plane dislocations appear during the transformation. These additional dislocations dominate the microstructure of the advancing martensite phase near the phase boundary. During the advance of the phase boundaries they can create or absorb stacking faults, depending on the time spend in the transformation path. External stresses can well be absorbed by these dislocations. Twin boundaries as expected for the accommodation of the martensite variants at low temperatures would move by the same basal plane dislocation mechanisms of the $18 \mathrm{R}$ stacking. This movement we find pinned by crossing dislocations, here of type A. Kustov et $a l .{ }^{31}$ used the term of "dislocation jamming" of the interface mobility when they analyzed the effect of plastic deformation and heat treatment on the athermal stabilization of the martensite phase. In our experiments the interface mobility between austenite and martensite is still not suppressed while the jamming mechanism appears to relate strongly to the twin boundaries between the martensitic variants.

Support from Marie-Curie RTN MULTIMAT (Contract No. MRTN-CT-2004-505226) is acknowledged. D.S. and H.I. thank R. Espinoza for support with the TEM sample preparations. X.M. acknowledges support from Comissionat per a Universitats i Recerca (CUR) del Departament d'Innovacio, Universitats I Empresa, de la Generalitat de Catalunya.
${ }^{1}$ J. Van Humbeeck, J. Alloys Compd. 355, 58 (2003).

${ }^{2}$ D. Z. Liu and D. Dunne, Scr. Mater. 48, 1611 (2003).

${ }^{3}$ Y. N. Wang, X. H. Chen, and H. M. Shen, Proceedings of the 9th intern. Conference on internal friction and ultrasonic attenuation in solids, 305, (1990).

${ }^{4}$ V. N. Belko, B. M. Darinski, V. S. Postniko, and I. M. Sharshak, Phys. Met. Metallogr. 27, 140 (1969).

${ }^{5}$ G. Guenin, M. Morin, P. F. Gobin, W. Dejonghe, and L. Delaey, Mater. Corros. 185, 573 (1973).

${ }^{6}$ T. Xiao, Metall. Trans. A 24, 1067 (1993).

${ }^{7}$ W. Dejonghe, R. De Batist, and L. Delaey, Scr. Metall. 10, 1125 (1976).

${ }^{8}$ J. S. Zhu, R. Schaller, and W. Benoit, Phys. Status Solidi A 108, 613 (1988)

${ }^{9}$ Z. L. Song, J. S. Zhu, Y. S. Qian, A. S. Aruna, Y. N. Wang, and C. Xie, Solid State Commun. 118, 257 (2001).

${ }^{10}$ G. Gremaud, J. E. Bidaux, and W. Benoit, Helv. Phys. Acta 60, 947 (1987).

${ }^{11}$ W. Cai, X. L. Lu, and L. C. Zhao, Mater. Sci. Eng., A 394, 78 (2005).

${ }^{12}$ J. X. Zhang, Z. H. Yang, and P. C. W. Fung, Phys. Rev. B 52, 278 (1995).

${ }^{13}$ C. L. Gong, F. S. Han, Z. Li, and M. P. Wang, Phys. Rev. B 70, 094103 (2004).

${ }^{14}$ Z. L. Song, S. Kishimoto, J. S. Zhu and Y. N. Wang, Solid State Commun. 139, 235 (2006); for phase diagram see also M. Jurado, T. Castan, L. Manosa, Antoni Planes, Josep Bassas, Xavier Alcobé, and Michel Morin, Philos. Mag. A 75, 1237 (1997).

${ }^{15}$ J. X. Zhang, Acta Mater. 47, 2125 (1999).

${ }^{16}$ R. J. Harrison, S. A. T. Redfern, and E. K. H. Salje, Phys. Rev. B 69, 144101 (2004); R. J. Harrison, S. A. T. Redfern, A. Buckley, and E. K. H. Salje, J. Appl. Phys. 95, 1706 (2004).

${ }^{17}$ E. K. H. Salje and H. Zhang, J. Phys.: Condens. Matter 21, 035901 (2009).

${ }^{18}$ A. V. Kityk, W. Schranz, P. Sondergeld, D. Havlik, E. K. H. Salje, and J. F. Scott, Phys. Rev. B 61, 946 (2000).
${ }^{19}$ E. Salje, B. Palosz, and B. Wruck, J. Phys. C 20, 4077 (1987).

${ }^{20}$ R. J. Harrison and S A T. Redfern, Phys. Earth Planet. Inter. 134, 253 (2002); power law decay was observed in L. Dong, D. S. Stone, and R. S. Lakes, Phys. Status Solidi B 11, 2422 (2008); and some theoretical underpinning was given by R. B. PerezSaez, V. Recarte, M. L. No, and J. San Juan, Phys. Rev. B 57, 5684 (1998).

${ }^{21}$ E. K. H. Salje, Phys. Chem. Miner. 35, 321 (2008).

${ }^{22}$ R. J. Harrison, S. A. T. Redfern, and J. Street, Am. Mineral. 88, 574 (2003)

${ }^{23}$ R. J. Harrison, S. A. T. Redfern, and U. Bismayer, Miner. Mag. 68, 839 (2004).

${ }^{24}$ N. Suresh and U. Ramamurty, Smart Mater. Struct. 14, N47, (2005).

${ }^{25}$ M. L. Castro and R. Romero, Mater. Sci. Eng., A 287, 66 (2000).

${ }^{26}$ A. Planes, L. Mañosa, D. Rios-Jara, and J. Ortin, Phys. Rev. B 45, 7633 (1992).

${ }^{27}$ C. H. Gonzalez, C. J. De Araujo, N. F. Quadros, G. Guenin, and M. Morin, Mater. Sci. Eng., A 378, 253 (2004).

${ }^{28}$ T. Xiao and G. P. Johari, Metall. Trans. A 26, 721 (1995).

${ }^{29}$ T. Xiao, G. P. Johari, and C. Mai, Metall. Trans. A 24, 2743 (1993).

${ }^{30}$ J. I. Perez-Landazabal, V. Recarte, D. S. Agosta, V. SanchezAlarcos, and R. G. Leisure, Phys. Rev. B 73, 224101 (2006).

${ }^{31}$ A. Kustov, J. Pons, E. Cesari, M. Morin, and J. Van Humbeeck, Mater. Sci. Eng., A 373, 328 (2004).

${ }^{32}$ K. Otsuka, T. Nakamura, and K. Shimizu, Trans. Jpn. Inst. Met. 15, 200 (1974).

${ }^{33}$ J. W. Brooks, M. H. Loretto, and R. E. Smallman, Acta Mater. 27, 1829 (1979).

${ }^{34}$ F. C. Lovey, G. Van Tandeloo, J. Van Landuyt, L. Delaey, and S. Amelinckx, Phys. Status Solidi A 86, 553 (1984).

${ }^{35}$ P. L. Rodriguez, A. Cuniberti, R. Romero, and F. C. Lovey, Scr. Metall. Mater. 27, 1133 (1992).

${ }^{36}$ P. L. Rodriguez, A. M. Condo, and F. C. Lovey, Phys. Status Solidi B 197, 279 (1996). 